\title{
Diabetes Mellitus Risk Assessment Among Egyptians Versus Saudi Arabians Nursing Employees: Comparative Study
}

\author{
Hassnaa Eid Shaban Mosa ${ }^{{ }^{*}} \quad$ Ayat Masoud Omar ${ }^{2}$ Shahenda Ateyat Allah Salih² \\ Eman Fawzy El Azab² \\ 1.Lecturer in Medical Surgical Nursing Department, Faculty of Nursing, Menoufia University, Egypt \\ 2.Assistant Professors, College of Applied Medical Sciences, Jouf University. KSA
}

\begin{abstract}
Purpose: Diabetes mellitus is the uppermost vital public health challenges in the world. More than 150 million adults are affected and the total number is predicted to double in the next 25 years. The present study aiming to assess the diabetes mellitus risk among Egyptians versus Saudi Arabian's nursing employees. Methods: A convenient sample of 150 employees from each country were collected. The study was conducted at faculties of Nursing at Menoufia University, Egypt and Jouf University, Kingdom of Saudia Arabia. Two tools were utilized for data collection, self-administered questionnaire and self-risk assessment screening score. Results: Both groups had a good total knowledge score ( $74 \%$ of Egyptians vs. $58 \%$ of Saudi Arabians) with a statistically significant difference. More than half of Egyptians had negative attitude compared to nearly half of Saudi Arabians who had a positive attitude. Nearly two-thirds of Egyptians had good practice vs. $44 \%$ of Saudi Arabians who had a bad practice. More than two-thirds of Egyptians vs. more than three-fourths of Saudi Arabians were scored high risk for diabetes mellitus. Conclusion: Based on the findings, there were statistically significant differences among Egyptians versus Saudi Arabian's nursing employees in total knowledge, practice and attitude scores of diabetes mellitus. Total risk assessment screening score of diabetes mellitus has shown a statistically significant difference between both groups. Recommendations: Large-scale awareness programs should be implemented after identifying the appropriate means to spread the message to the common population about all aspects of diabetes.
\end{abstract}

Keywords: Diabetes mellitus, Knowledge, Practices, Attitude, Risk assessment.

DOI: $10.7176 / \mathrm{JHMN} / 59-04$

\section{Introduction}

The international occurrence of diabetes mellitus is growing rapidly providing a disturbing sign and risk factor to global health. Diabetes mellitus is distinguished by chronic hyperglycemia and impaired carbohydrates, proteins and lipids metabolism caused by complete or partial insufficiency of insulin secretion and/or insulin action. There are two crucial forms of diabetes, type 1 (insulin-dependent diabetes mellitus) and non-insulin-dependent diabetes mellitus (type 2) (Yanlin et al., 2014). The extreme occurrence of diabetes overall is predicted to occur in North Africa due to fast economic development, civilization and changes in lifestyle patterns in the region (Whiting et al., 2011). World Health Organization estimated 6.7 million diabetic patients in Egypt, it has been predestined that, the number of diabetic patient in Egypt will be 8.6 million by the year 2030. This number will make Egypt tenth largest population of diabetics in the world with a significant effect on morbidity, mortality and health care resources. While, Saudia Arabia is the second highest ranking in the Middle East and is the seventh in the world for the rate of diabetes with the maximum prevalence which equals $23.9 \%$ (Naeem, 2015).

Diabetes happens due to damage in beta-cell function which is basically the responsible for the progression from normoglycemia to hyperglycemia, insulin resistance pre-exists beta-cell dysfunction and plays the main role in the pathogenesis of type 2 diabetes (Shulman, 2014). Although diabetic patients with type2 are usually independent of exogenous insulin, they may require it when the level of blood glucose is not completely controlled with diet or with oral hypoglycemic drugs. Moreover, diabetic patients are often escorted by complications, such as diabetic neuropathy, cardiovascular diseases, retinopathy and nephropathy. Diabetes and its complications depress the quality of people's lives and produce huge social and economic problems (Zhao et al., 2011).

Knowledge, attitude and practice (KAP) concerning diabetes differ significantly depending on socioeconomic environments, habits and cultural beliefs which affecting positively or negatively in disease progression. Understanding these variables is important in designing preventive and management strategies for diabetes (Perez \& Cha, 2017, Herath et al., 2017). All KAP components are interconnected and dependent on each other, if the level of one component is higher, the other two factors should be affected consequently (Zhao et al., 2011).

Diabetes mellitus risk assessment screening is one of the most important methods for early detection of undiagnosed diabetic and pre-diabetic/borderline diabetic personnel which should be implemented periodically and continuously by health personnel (Kassahun \& Mekonen, 2017).

\subsection{Significance of the study}

Every year about 4.9 million people are killed by diabetes. This consumes the nations' health care budget unless 
interventions are created through community awareness. Levels of awareness and factors associated with knowledge, practice, and attitude towards diabetes mellitus are essential to delivering appropriate diabetes information to the community. As researchers possess the community aware of diabetes, the community can participate in the prevention and controlling diabetes.

\subsection{Aim of the study: This study aims to}

Assess the diabetes mellitus risk among Egyptians versus Saudi Arabian’s nursing employees.

\subsection{Research Questions:}

1. Is there a statistically significant difference between both groups in the level of knowledge, attitude, and practice, in relation to diabetes mellitus?

2. Is there a statistically significant difference in the risk of diabetes mellitus between both groups?

\section{Methodology}

2.1 Design: A non-experimental descriptive research design was utilized in this study.

2.2 Setting: The study was carried out at the Faculties of Nursing, Menoufia University, Egypt and Jouf University, Kingdom of Saudia Arabia.

2.3 Sample: A convenient sample of 300 employees (150 Egyptians and 150 Saudi Arabians).

\section{Subjects had been selected according to the following inclusion criteria:}

1. Both sexes, free from psychiatric problem.

2. Able to cooperate and communicate

3. Willing to participate in the study

\section{Exclusion criteria:}

1. Diabetic employees

2. Refuse to participate

Data collection tools: two tools were utilized for data collection in this study.

Tool I: A self- administered questionnaire: designed by the researchers and validated to collect the necessary data. It based on an intensive review of related literature and included two parts as follow:

Part one: Employee's bio-demographic data which including two sections as the following:

Section one: Employee's personal data which included six questions about employee's education, marital status, nature of work, residence, income status, and self-reported personality nature.

Section two: Employee's medical data included three questions about the presence of other diseases, smoking, and previous delivery of a macrosomic baby.

Part two: Employee's knowledge, attitude and practices assessment questionnaire which was constructed by the researcher to assess the employee's knowledge, practice and attitude about diabetes mellitus based on an intensive recent review of the literature and similar studies conducted elsewhere. The questionnaire divided into three sections as the following.

Section one: Employee's knowledge assessment sheet which consisted of five questions to assess the employee's knowledge about diabetes mellitus.

Scoring system: It is measured by calculating the mean score of the five items with three-point Likert's scale( complete correct answer (two points), the incomplete correct answer (one point) and wrong answer(zero point). All employee's answers about knowledge questions were computed to obtain total mean scores and categorized as poor knowledge if the employee scored $\leq 50 \%$ or fair knowledge if the employee scored $50-65 \%$ and good knowledge if the employee scored $>65 \%$ of total knowledge score.

Section two: Employee's attitude assessment sheet which consisted of nine questions to assess the way by which the employees thinks and behaves toward diabetes mellitus.

Scoring system: It is measured by with three-point Likert's scale (yes (two points), to some extent (one point) and no (zero point). All employee's answers were computed to obtain total scores then, the mean score was calculated to categorize as having a negative attitude if the employee's scored $\leq 50 \%$ or ordinary attitude if the employee's scored $50-65 \%$ and positive attitude if the employee's scored $>65 \%$ of the total attitude score.

Section three: Employee's practices assessment sheet: It consisted of eleven questions to assess the habitual employee's involvement in their life which affects developing diabetes mellitus.

Scoring system: It is measured by three-point Likert's scale (always (two points), sometimes (one point) and never (zero point)). All employee's answers were computed to obtain total mean scores and categorized as bad practice if the employees scored $\leq 50 \%$ or accepted practice if the employees scored $50-65 \%$ and good practice if the employees scored $>65 \%$ of the total practice score.

Tool II: A self- risk assessment screening score: Adapted by the researcher from Bang et al., 2009. It included six 
questions as age, sex, family history of diabetes, the presence of hypertension, body mass index and physical activity. The total score of this score was ten.

\section{Scoring system:}

1. If the total score was $<5$ the employee is at low risk for diabetes mellitus.

2. If the total score was $\geq 5$ the employee is at high risk for diabetes mellitus.

\section{Content Validity:}

It was established by a panel of 5 experts in Medical-Surgical Nursing who reviewed the tools for clarity, relevance, comprehensiveness, understanding, applicability, and easiness.

\section{Reliability:}

Reliability of the tools was done by test-retest method and Pearson correlation coefficient formula to ascertain the relevance and consistency of the tools and measure its items. It was 0.89 for the first tool and 0.93 for the second tool.

\subsection{Pilot study:}

The pilot study carried out for $10 \%$ of employees in each country to test the feasibility of tools and time required to be applied. A simple modification was done for some items of the data collection tools.

\section{Ethical consideration:}

1. The protocol was approved by pertinent research and ethical committees.

2. Informed consent was taken from every employee before inclusion in the study and after the explanation of the aims of the study.

3. Employees assured that all data will be highly confidential, anonymity will be also assured through assigning a code number for each participant instead of names to save their privacy.

4. Data were available only to the researchers and the employees.

5. The researchers confirmed that participation in the current study was entirely voluntary.

\subsection{Procedure:}

1. Before the conduction of the pilot study, official permission was obtained from the dean of each faculty in each country

2. Data collection divided over a period of two months from the beginning of April to the end of May 2018 in KSA then the researchers collect data from Egypt over a period of two months from the beginning of June to the end of July 2018.

3. A self-administered questionnaire in Arabic form was distributed among employees. After recruitment in the study, the filling up the questionnaire took around 30 to 45 minutes.

4. The researchers initiated data collection by interviewing each employee individually in their administrative offices.

5. Bio-demographic data were assessed by using part one of tool I.

6. Body mass index was calculated by dividing weight/kilogram by square of height/ meter. Then it was classified according to Bang et al., 2009 as the following:

- If body mass index $\geq 40 \mathrm{Kg} / \mathrm{M}^{2}$ the employee scored 3(extremely obese).

- If $30 \leq$ body mass index $<40 \mathrm{Kg} / \mathrm{M}^{2}$ the employee scored 2 (obese).

- If $25 \leq$ body mass index $<30 \mathrm{Kg} / \mathrm{M}^{2}$ the employee scored 1 (overweight).

- If body mass index $<25 \mathrm{Kg} / \mathrm{M}^{2}$ the employee scored 0 (not obese or overweight).

7. Each employee knowledge, attitude, and practice regarding diabetes mellitus were assessed using part two of tool I.

8. Diabetes mellitus risk assessment was assessed using Bang et al., 2009 screening risk score items.

9. All obtained data were analyzed to assess the diabetes mellitus risk among Egyptians versus Saudi Arabian's nursing employees.

\subsection{Statistical methodology}

The collecting data were analyzed by SPSS (statistical package for the social science software) version 22 on IBM compatible computer. For qualitative analysis, it was explained as number and percentage and analyzed by stratifying the chi-square test. If the expected values in one or more of the cells in a $2 \times 2$ tables were less than 5, Fisher exact test was used. All these tests were used as tests of significance at $\mathrm{P}<0.05$ (Dawson \& Trapp, 2001).

\section{Results}

Table (1) revealed that, there were statistically significant difference regarding the nature of work, residence, income status and self-reported personality nature between both groups as more than two thirds of Egyptians versus the majority of Saudi Arabians had mental nature of work and more than two thirds of Egyptians had nervous personality nature vs. about half of Saudi Arabians whom had quiet personality nature. Furthermore, the 
majority of Egyptians had income equal to the expense while about half of Saudi Arabians had income more than the expense. In contrast, no statistically significant differences were reported between both groups regarding education and marital status.

Table 1: Distribution of personal data of both groups.

\begin{tabular}{|c|c|c|c|c|c|c|}
\hline \multirow{3}{*}{ Personal data } & \multicolumn{4}{|c|}{ Groups } & \multirow{3}{*}{$\mathbf{X}^{2}$} & \multirow{3}{*}{ P value } \\
\hline & \multicolumn{2}{|c|}{ Egyptian } & \multicolumn{2}{|c|}{ Saudi Arabians } & & \\
\hline & $\mathrm{N}=150$ & $\%$ & $\mathrm{~N}=\mathbf{1 5 0}$ & $\%$ & & \\
\hline Education level: & & & & & \multirow{5}{*}{5.42} & \\
\hline Illiterate & 4 & $2.7 \%$ & 6 & $4 \%$ & & \\
\hline Primary \& preparatory & 18 & $12 \%$ & 14 & $9.3 \%$ & & 0.24 \\
\hline Secondary & 36 & $24 \%$ & 28 & $18.7 \%$ & & $>0.05$ \\
\hline University \& above & 92 & $61.3 \%$ & 102 & $68 \%$ & & \\
\hline \multicolumn{7}{|l|}{ Marital status: } \\
\hline Single & 74 & $49.3 \%$ & 76 & $50.7 \%$ & \multirow{3}{*}{8.26} & 0.12 \\
\hline Married & 51 & $34 \%$ & 50 & $33.3 \%$ & & $>0.05$ \\
\hline Divorced & 25 & $16.7 \%$ & 24 & $16 \%$ & & \\
\hline \multicolumn{7}{|l|}{ Nature of work: } \\
\hline Muscular work & 36 & $24 \%$ & 9 & $6 \%$ & \multirow{3}{*}{$11.743^{\mathrm{a}}$} & \\
\hline Mental work & 97 & $64.7 \%$ & 135 & $90 \%$ & & 0.003 \\
\hline Muscular \& mental work & 17 & $11.3 \%$ & 6 & $4 \%$ & & $<0.01$ \\
\hline \multicolumn{7}{|l|}{ Residence: } \\
\hline Urban & 97 & $65 \%$ & 135 & $90 \%$ & \multirow{2}{*}{$16.783^{\mathrm{a}}$} & \multirow{2}{*}{0.001} \\
\hline Rural & 53 & $36 \%$ & 15 & $10 \%$ & & \\
\hline \multicolumn{7}{|l|}{ Income status: } \\
\hline More than expense & 0 & $0.0 \%$ & 69 & $46 \%$ & \multirow{3}{*}{$80.581^{\mathrm{a}}$} & \multirow{3}{*}{0.001} \\
\hline Equal to expense & 136 & $90.7 \%$ & 63 & $42 \%$ & & \\
\hline Less than expense & 14 & $9.3 \%$ & 18 & $12 \%$ & & \\
\hline \multicolumn{7}{|c|}{ Self-reported personality nature: } \\
\hline Nervous & 102 & $68 \%$ & 45 & $30 \%$ & \multirow{3}{*}{$14.201^{\mathrm{a}}$} & \multirow{3}{*}{0.001} \\
\hline Medium & 45 & $30 \%$ & 36 & $24 \%$ & & \\
\hline Quiet & 3 & $2 \%$ & 69 & $46 \%$ & & \\
\hline
\end{tabular}

\section{$\left({ }^{a}\right)$ : Fisher Exact Test.}

Table (2) shown the distribution of medical data of both groups. It clarified that there was no statistically significant difference between both groups except for the history of smoking and previous delivery of macrosomic babies. The majority of Egyptians weren't smokers versus more than three-fourths of Saudi Arabians who were smokers. More than half of Egyptian women hadn't previous delivery of a macrosomic baby while two-thirds of Saudi Arabians women had previous delivery of a macrosomic baby.

Table 2: Distribution of medical data of both groups.

\begin{tabular}{|c|c|c|c|c|c|c|}
\hline \multirow{3}{*}{ Medical data } & \multicolumn{4}{|c|}{ Groups } & \multirow{3}{*}{$\mathbf{X}^{2}$} & \multirow{3}{*}{ P value } \\
\hline & \multicolumn{2}{|c|}{ Egyptians } & \multicolumn{2}{|c|}{ Saudi Arabians } & & \\
\hline & $\mathrm{N}=150$ & $\%$ & $\mathbf{N}=\mathbf{1 5 0}$ & $\%$ & & \\
\hline \multicolumn{7}{|c|}{ Presence of other chronic illnesses: } \\
\hline Yes & 44 & $29.3 \%$ & 24 & $16 \%$ & \multirow{3}{*}{$3.840^{\mathrm{a}}$} & \multirow{3}{*}{0.147} \\
\hline No & 90 & $60 \%$ & 102 & $68 \%$ & & \\
\hline I don't know & 16 & $10.7 \%$ & 24 & $16 \%$ & & \\
\hline \multicolumn{7}{|c|}{ Types of present chronic illnesses: } \\
\hline Hypertension & 20 & $45.5 \%$ & 6 & $25 \%$ & \multirow{3}{*}{$1.370^{\mathrm{a}}$} & \multirow{3}{*}{0.504} \\
\hline High blood lipids & 6 & $13.6 \%$ & 6 & $25 \%$ & & \\
\hline Others $\left({ }^{b}\right)$ & 18 & $40.9 \%$ & 12 & $50 \%$ & & \\
\hline \multicolumn{7}{|l|}{ Smoking: } \\
\hline Yes & 6 & $4 \%$ & 126 & $84 \%$ & \multirow{2}{*}{$10.180^{\mathrm{a}}$} & \multirow{2}{*}{0.003} \\
\hline No & 144 & $96 \%$ & 24 & $16 \%$ & & \\
\hline \multicolumn{7}{|c|}{ Women with previous delivery of macrosomic baby: } \\
\hline Yes & 34 & $37.7 \%$ & 25 & $59.5 \%$ & \multirow{3}{*}{9.6} & \multirow{3}{*}{0.03} \\
\hline No & 50 & $55.6 \%$ & 10 & $23.8 \%$ & & \\
\hline I do not know & 6 & $6.7 \%$ & 7 & $16.7 \%$ & & \\
\hline
\end{tabular}

(a): Fisher Exact Test.

$\left({ }^{b}\right)$ : other diseases as (stomach ulcer, osteoarthritis and hyperthyroidism) 
Table (3) cleared up that; both groups had good total knowledge score (74\% of Egyptians vs. 58\% of Saudi Arabians) with a statistically significant difference. Meanwhile, employee's knowledge regarding parts of diabetes mellitus risk factors and complications revealed a high statistically significant difference as nearly three-quarters of Egyptians had the complete correct answer vs. nearly two-thirds of Saudi Arabians.

Table 3: Distribution of knowledge regarding diabetes mellitus of both groups.

\begin{tabular}{|c|c|c|c|c|c|c|}
\hline \multirow{3}{*}{ Items } & \multicolumn{4}{|c|}{ Groups } & \multirow{3}{*}{$\mathbf{X}^{2}$} & \multirow{3}{*}{ P Value } \\
\hline & \multicolumn{2}{|c|}{ Egyptians } & \multicolumn{2}{|c|}{ Saudi Arabians } & & \\
\hline & $N=150$ & $\%$ & $\mathbf{N}=\mathbf{1 5 0}$ & $\%$ & & \\
\hline \multicolumn{7}{|l|}{ Definition: } \\
\hline Wrong answer & 38 & $25.3 \%$ & 36 & $24 \%$ & & \\
\hline Incomplete correct answer & 13 & $8.7 \%$ & 27 & $18 \%$ & $3.371^{\mathrm{a}}$ & 0.185 \\
\hline Complete correct answer & 99 & $66 \%$ & 87 & $58 \%$ & & \\
\hline \multicolumn{7}{|l|}{ Risk factors: } \\
\hline Wrong answer & 32 & $21.3 \%$ & 30 & $20 \%$ & & \\
\hline Incompletely correct answer & 4 & $2.7 \%$ & 36 & $24.5 \%$ & $24.169^{\mathrm{a}}$ & 0.000 \\
\hline Complete correct answer & 114 & $76 \%$ & 84 & $56.1 \%$ & & \\
\hline \multicolumn{7}{|l|}{ Signs and symptoms: } \\
\hline Wrong answer & 20 & $13.3 \%$ & 18 & $12 \%$ & & \\
\hline Incomplete correct answer & 16 & $10.7 \%$ & 24 & $16 \%$ & $1.020^{\mathrm{a}}$ & 0.600 \\
\hline Complete correct answer & 114 & $76 \%$ & 108 & $72 \%$ & & \\
\hline \multicolumn{7}{|l|}{ Treatment modalities: } \\
\hline Wrong answer & 26 & $17.3 \%$ & 24 & $16 \%$ & & \\
\hline Incomplete correct answer & 10 & $6.7 \%$ & 30 & $20 \%$ & $7.446^{\mathrm{a}}$ & 0.024 \\
\hline Completely correct answer & 114 & $76.0 \%$ & 96 & $64 \%$ & & \\
\hline \multicolumn{7}{|l|}{ Complications of diabetes: } \\
\hline Wrong answer & 40 & $26.7 \%$ & 39 & $26 \%$ & & \\
\hline Incomplete correct answer & 0 & $0.0 \%$ & 27 & $18 \%$ & $28.639^{\mathrm{a}}$ & 0.000 \\
\hline Complete correct answer & 110 & $73.3 \%$ & 84 & $56 \%$ & & \\
\hline \multicolumn{7}{|l|}{ Total knowledge score (10): } \\
\hline Poor knowledge score $\leq 50 \%$ & 36 & $24 \%$ & 57 & $38 \%$ & $11.044^{\mathrm{a}}$ & 0.011 \\
\hline Fair knowledge score $>50<65$ & 3 & $2 \%$ & 6 & $4 \%$ & & \\
\hline Good knowledge score $\geq 65 \%$ & 111 & $74 \%$ & 87 & $58 \%$ & & \\
\hline
\end{tabular}

\section{${ }^{(a)}$ : Fisher Exact Test}

Table (4) illustrated that there was a statistically significant difference regarding the total score of the employee's attitude toward diabetes mellitus between both groups. More than half of Egyptians employees had negative attitude compared to nearly half of Saudi Arabians were had a positive attitude. 
Table 4: Distribution of employee's attitude regarding diabetes mellitus of both groups.

\begin{tabular}{|c|c|c|c|c|c|c|}
\hline \multirow[b]{3}{*}{ Attitude questions } & \multicolumn{4}{|c|}{ Groups } & \multirow{3}{*}{$\mathbf{X}^{2}$} & \multirow{3}{*}{ P value } \\
\hline & \multicolumn{2}{|c|}{ Egyptians } & \multicolumn{2}{|c|}{ Saudi Arabians } & & \\
\hline & $\mathrm{N}=\mathbf{1 5 0}$ & $\%$ & $\mathbf{N}=\mathbf{1 5 0}$ & $\%$ & & \\
\hline \multicolumn{7}{|c|}{ It is a social stigma to be diagnosed with diabetes mellitus: } \\
\hline No & 22 & $14.7 \%$ & 24 & $16 \%$ & \multirow{3}{*}{$1.474^{\mathrm{a}}$} & \multirow{3}{*}{0.479} \\
\hline To some extent & 64 & $42.7 \%$ & 63 & $42 \%$ & & \\
\hline Yes & 64 & $42.7 \%$ & 63 & $42 \%$ & & \\
\hline \multicolumn{7}{|c|}{ Diabetes complications will not occur if the blood glucose level is well controlled: } \\
\hline No & 18 & $12 \%$ & 18 & $12 \%$ & \multirow{3}{*}{$10.695^{\mathrm{a}}$} & \multirow{3}{*}{0.005} \\
\hline To some extent & 30 & $20 \%$ & 66 & $44 \%$ & & \\
\hline Yes & 102 & $68 \%$ & 66 & $44 \%$ & & \\
\hline \multicolumn{7}{|c|}{ Nervousness and continuous anxiety are major risk factors to develop the diseases: } \\
\hline No & 66 & $44 \%$ & 63 & $42 \%$ & \multirow{3}{*}{$22.806^{\mathrm{a}}$} & \multirow{3}{*}{0.000} \\
\hline To some extent & 18 & $12 \%$ & 63 & $42 \%$ & & \\
\hline Yes & 66 & $44 \%$ & 24 & $16 \%$ & & \\
\hline \multicolumn{7}{|c|}{ Diabetes affects badly on all person's life style patterns: } \\
\hline No & 38 & $25.3 \%$ & 51 & $34 \%$ & \multirow{3}{*}{$1.687^{\mathrm{a}}$} & \multirow{3}{*}{0.430} \\
\hline To some extent & 45 & $30 \%$ & 45 & $30 \%$ & & \\
\hline Yes & 67 & $44.7 \%$ & 54 & $36 \%$ & & \\
\hline \multicolumn{7}{|c|}{ Type I diabetes mellitus equal type II in its severity: } \\
\hline No & 97 & $64.7 \%$ & 36 & $24 \%$ & \multirow{3}{*}{$32.088^{\mathrm{a}}$} & \multirow{3}{*}{0.000} \\
\hline To some extent & 25 & $16.7 \%$ & 81 & $54 \%$ & & \\
\hline Yes & 28 & $18.7 \%$ & 33 & $22 \%$ & & \\
\hline \multicolumn{7}{|c|}{ Extreme control is a logical action only for people with juvenile diabetes: } \\
\hline No & 50 & $33.3 \%$ & 27 & $18 \%$ & \multirow{3}{*}{$36.153^{\mathrm{a}}$} & \multirow{3}{*}{0.000} \\
\hline To some extent & 15 & $10 \%$ & 72 & $48 \%$ & & \\
\hline Yes & 85 & $56.6 \%$ & 51 & $34 \%$ & & \\
\hline The disease severity with or & ent is th & same as $\mathrm{i}$ & ulin inje & ion treatn & nt: & \\
\hline No & 83 & $56 \%$ & 60 & $40 \%$ & & \\
\hline To some extent & 25 & $17 \%$ & 57 & $38 \%$ & $9.814^{\mathrm{a}}$ & 0.007 \\
\hline Yes & 42 & $28 \%$ & 33 & $220 \%$ & & \\
\hline Diabetes will occurs anywhe & cially if $t$ & re is a p & history & diabetes: & & \\
\hline No & 30 & $20 \%$ & 18 & $12 \%$ & & \\
\hline To some extent & 13 & $9 \%$ & 57 & $38 \%$ & $23.259^{\mathrm{a}}$ & 0.000 \\
\hline Yes & 107 & $72 \%$ & 75 & $50 \%$ & & \\
\hline Herbal remedies are better & lication $\mathrm{t}$ & diabete & & & & \\
\hline No & 50 & $33.6 \%$ & 51 & $34 \%$ & $7.408^{a}$ & 0.025 \\
\hline To some extent & 60 & $40 \%$ & 87 & $58 \%$ & & \\
\hline Yes & 40 & $26.7 \%$ & 12 & $8 \%$ & & \\
\hline Total attitude score (18): & & & & & & \\
\hline Positive attitude $>65 \%$ & 22 & 14.7 & 72 & $48 \%$ & & \\
\hline Ordinary attitude $>50 \leq 65$ & 52 & 34.7 & 48 & $32 \%$ & $10.04^{\mathrm{a}}$ & 0.01 \\
\hline Negative attitude $\leq 50 \%$ & 76 & 50.4 & 30 & $20 \%$ & & \\
\hline
\end{tabular}

\section{(a): Fisher Exact Test}

Table (5) presented the score of employee's practice regarding diabetes mellitus between both groups. It showed that there was a statistically significant difference between both groups. Nearly two-thirds of Egyptians had good practice vs. $44 \%$ of Saudi Arabians who had a bad practice. 
Table 5: Distribution of employee's practices regarding diabetes mellitus of both groups.

\begin{tabular}{|c|c|c|c|c|c|c|}
\hline \multirow{3}{*}{ Practice questions } & \multicolumn{4}{|c|}{ Groups } & \multirow{3}{*}{$\mathbf{X}^{2}$} & \multirow{3}{*}{ P Value } \\
\hline & \multicolumn{2}{|c|}{ Egyptians } & \multicolumn{2}{|c|}{ Saudi Arabians } & & \\
\hline & $\mathbf{N}(150)$ & $\%$ & $\mathbf{N}(150)$ & $\%$ & & \\
\hline \multicolumn{7}{|c|}{ Consumption > 3 meals / day: } \\
\hline Never & 32 & $21.3 \%$ & 33 & $22 \%$ & \multirow{3}{*}{$0.946^{\mathrm{a}}$} & \multirow{3}{*}{0.623} \\
\hline Some times & 62 & $41.3 \%$ & 51 & $34 \%$ & & \\
\hline Always & 56 & $37.3 \%$ & 66 & $44 \%$ & & \\
\hline \multicolumn{7}{|c|}{ Dependence on fast $\&$ fatty food: } \\
\hline Never & 23 & $15.3 \%$ & 57 & $38 \%$ & \multirow{3}{*}{$12.744^{\mathrm{a}}$} & \multirow{3}{*}{0.002} \\
\hline Some times & 59 & $39.3 \%$ & 54 & $36 \%$ & & \\
\hline Always & 68 & $45.3 \%$ & 39 & $26 \%$ & & \\
\hline \multicolumn{7}{|c|}{ Consumption of soft highly sugar drinks: } \\
\hline Never & 50 & $33.3 \%$ & 36 & $24 \%$ & \multirow{3}{*}{$2.874^{\mathrm{a}}$} & \multirow{3}{*}{0.238} \\
\hline Some times & 80 & $53.3 \%$ & 81 & $54 \%$ & & \\
\hline Always & 20 & $13.3 \%$ & 33 & $22 \%$ & & \\
\hline \multicolumn{7}{|c|}{ Consumption of fried than grilled food: } \\
\hline Never & 42 & $28 \%$ & 51 & $34 \%$ & \multirow{3}{*}{$6.835^{\mathrm{a}}$} & \multirow{3}{*}{0.033} \\
\hline Some times & 62 & $41.3 \%$ & 81 & $54 \%$ & & \\
\hline Always & 46 & $30.7 \%$ & 18 & $12 \%$ & & \\
\hline \multicolumn{7}{|c|}{ Consumption vegetables and fruits of different colors: } \\
\hline Never & 42 & $28 \%$ & 36 & $24 \%$ & \multirow{3}{*}{$19.696^{\mathrm{a}}$} & \\
\hline Some times & 18 & $12 \%$ & 60 & $40 \%$ & & 0.000 \\
\hline Always & 90 & $60 \%$ & 54 & $36 \%$ & & \\
\hline Consumption of high carb & ate diet: & & & & & \\
\hline Never & 36 & $24 \%$ & 48 & $32 \%$ & & \\
\hline Some times & 72 & $48 \%$ & 84 & $56 \%$ & $5.403^{\mathrm{a}}$ & 0.067 \\
\hline Always & 42 & $28 \%$ & 18 & $12 \%$ & & \\
\hline Eat just before sleeping: & & & & & & \\
\hline Never & 36 & $24 \%$ & 27 & $18 \%$ & & \\
\hline Some times & 70 & $46.7 \%$ & 78 & $52 \%$ & $0.839^{\mathrm{a}}$ & 0.657 \\
\hline Always & 44 & $29.3 \%$ & 45 & $30 \%$ & & \\
\hline Highly consumption of foc & a emotio & l instabil & & & & \\
\hline Never & 22 & $14.7 \%$ & 15 & $10 \%$ & & \\
\hline Some times & 93 & $62.0 \%$ & 99 & $66 \%$ & $0.707^{\mathrm{a}}$ & 0.702 \\
\hline Always & 35 & $23.3 \%$ & 36 & $24 \%$ & & \\
\hline Chewing slowly & & & & & & \\
\hline Never & 46 & $30.7 \%$ & 45 & $30 \%$ & & \\
\hline Some times & 48 & $32 \%$ & 66 & $44 \%$ & $2.944^{\mathrm{a}}$ & 0.229 \\
\hline Always & 56 & $37.3 \%$ & 39 & $26 \%$ & & \\
\hline Monitoring glucose level $\mathbf{r}$ & ly especia & y with po & ive famil & history & & \\
\hline Never & 23 & $15.3 \%$ & 42 & $28.0 \%$ & & \\
\hline Some times & 27 & $18.0 \%$ & 42 & $28.0 \%$ & $8.240^{\mathrm{a}}$ & 0.016 \\
\hline Always & 100 & $66.7 \%$ & 66 & $44.0 \%$ & & \\
\hline Sleep $<8$ hours / day & & & & & & \\
\hline Never & 27 & $18 \%$ & 54 & $36 \%$ & & \\
\hline Some times & 68 & $45.3 \%$ & 57 & $38 \%$ & $7.119^{\mathrm{a}}$ & 0.028 \\
\hline Always & 55 & $36.7 \%$ & 39 & $26 \%$ & & \\
\hline Total practice score (22) & & & & & & \\
\hline Good practice $>65 \%$ & 89 & $59.3 \%$ & 54 & $36 \%$ & & \\
\hline Accepted practice $>50 \leq 65$ & 18 & $12 \%$ & 30 & $20 \%$ & 8.02 & 0.02 \\
\hline Bad practice $\leq 50 \%$ & 43 & $28.7 \%$ & 66 & $44 \%$ & & \\
\hline
\end{tabular}

\section{(a): Fisher Exact Test}

Table (6) clarified that there was a significant difference between both groups in relation to age, gender and the positive family history of diabetes mellitus. On the other hand, there were no significant differences between the two groups related to body mass index, the presence of hypertension and physical activity. 
Table 6: Distribution of risk assessment score of both groups.

\begin{tabular}{|c|c|c|c|c|c|c|c|}
\hline \multicolumn{2}{|c|}{ Risk assessment score } & \multicolumn{4}{|c|}{ Groups } & \multirow{3}{*}{$\mathbf{X}^{2}$} & \multirow{3}{*}{ P.value } \\
\hline \multirow[t]{2}{*}{ Items } & \multirow[t]{2}{*}{ Scoring system } & \multicolumn{2}{|c|}{ Egyptians } & \multicolumn{2}{|c|}{ Saudi Arabians } & & \\
\hline & & No(150) & $\%$ & No(150) & $\%$ & & \\
\hline \multicolumn{8}{|l|}{ Age: } \\
\hline$<40 Y$ & $(0)$ & 66 & 44 & 141 & 94 & \multirow{4}{*}{16.8} & \multirow{4}{*}{0.001} \\
\hline $40-49$ & (1) & 38 & 35.3 & 3 & 2 & & \\
\hline $50-59$ & (2) & 46 & 30.7 & 0 & 0 & & \\
\hline$\geq 60$ & (3) & 0 & 0 & 6 & 4 & & \\
\hline \multicolumn{8}{|l|}{ Gender: } \\
\hline Male & $(0)$ & 60 & 40 & 108 & 72 & \multirow{2}{*}{12.33} & \multirow{2}{*}{0.001} \\
\hline Female & (1) & 90 & 60 & 42 & 28 & & \\
\hline \multicolumn{8}{|c|}{ Positive family history of diabetes: } \\
\hline Present & $(1)$ & 87 & 58 & 57 & 38 & \multirow{2}{*}{21.2} & \multirow{2}{*}{0.001} \\
\hline Not present & $(0)$ & 63 & 42 & 93 & 62 & & \\
\hline \multicolumn{8}{|c|}{ Presence of hypertension: } \\
\hline Yes & $(1)$ & 87 & 58 & 93 & 62 & \multirow{2}{*}{6.3} & \multirow{2}{*}{0.052} \\
\hline No & $(0)$ & 63 & 42 & 57 & 38 & & \\
\hline \multicolumn{8}{|c|}{ Body mass index: } \\
\hline$\geq 40$ & (3) & 6 & 4 & 6 & 4 & \multirow{4}{*}{4.68} & \multirow{4}{*}{0.071} \\
\hline$\geq 30$ & (2) & 52 & 34.7 & 45 & 30 & & \\
\hline $25 \leq \mathrm{BMI}<30$ & (1) & 59 & 39.3 & 9 & 6 & & \\
\hline Normal & (0) & 33 & 22 & 90 & 60 & & \\
\hline \multicolumn{8}{|c|}{ Physical activity: } \\
\hline Yes & $(0)$ & 76 & 50.7 & 96 & 64 & \multirow{2}{*}{6.33} & \multirow{2}{*}{0.058} \\
\hline No & (1) & 74 & 49.3 & 54 & 36 & & \\
\hline
\end{tabular}

Figure (1) demonstrated that, there was a statistically significant difference $\left(\mathrm{X}^{2} 11.2-\mathrm{p} 0.032\right)$ in relation to diabetes mellitus risk assessment score between both groups as $32 \%$ of Egyptians vs. $21.3 \%$ of Saudi Arabians scored low risk for diabetes mellitus while more than two thirds of Egyptians vs. more than three froths of Saudi Arabians were scored as high risk for diabetes mellitus.

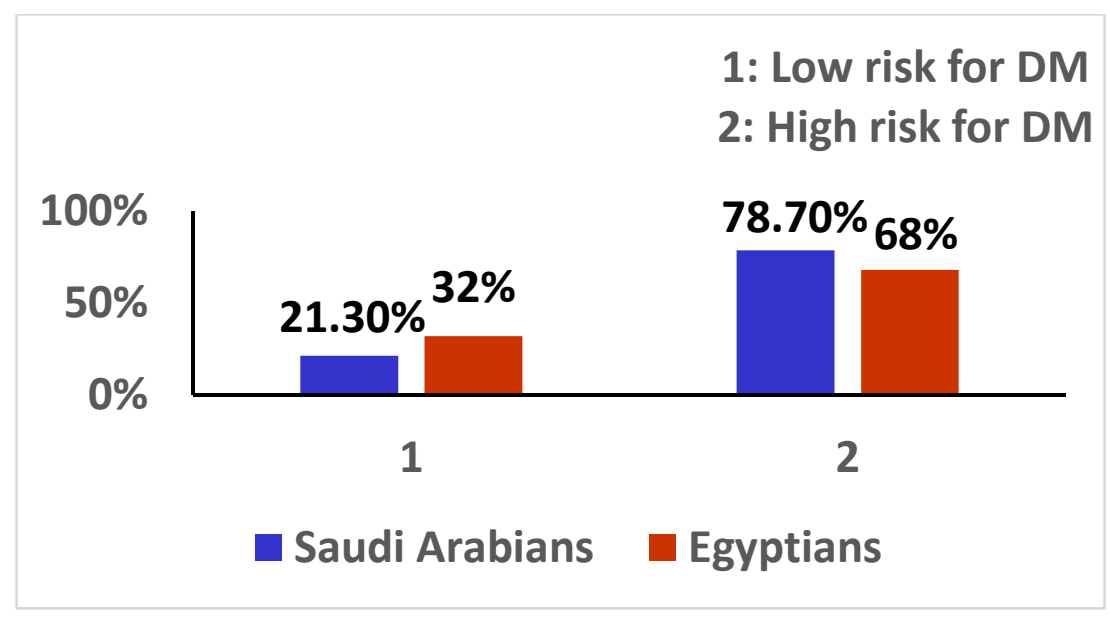

Figure 1: Total diabetes mellitus risk score of both groups.

\section{Discussion}

Diabetes mellitus (DM) is one of the foremost growing non-communicable disease intimidations to international public health. Inclinations in the occurrence of diabetes display a disproportionate rise in developing countries due to the present fast demographic conversions from old-style to additional civilian and westernized lifestyles (Yanling et al., 2014).

The current study revealed that most of Egyptians employees were not smokers while the majority of Saudi Arabian's employees were smokers, which is consider as an indicator that increases the risk score and the incidence among Saudi Arabian's employees. This is consistent with a previous study carried out about the health consequences of smoking by the Department of Health and Human Services (2014) and reported that, smoking was a main risk factor for many diseases especially diabetes mellitus. From the researcher point of view, elevated smoker's number among Saudi Arabians may be related to small age as the majority of them less than 40 year and 
elevated standard of living as the majority of them had income more than expense as evidenced in table six and one respectively.

As regards the total risk score of diabetes mellitus between both groups, the current study revealed a significant difference between both groups as more than two-thirds of Egyptians vs. more than three froths of Saudi Arabians were scored as high risk for diabetes mellitus (pre-diabetic/borderline diabetes or un-diagnosed diabetes that needs lab verification). From the researcher point of view, this is may be related to continuity of Saudi Arabians to take sweetened foods such as sweets, chocolates and dates served with coffee as a traditional habit in KSA. While in Egypt, this is may be related to the low socioeconomic status, which doesn't allow for healthy food choice.

Also, the age considered as an important risk factor that plays a significant role in this results. More than twothirds of Egyptians their age range between 40-59 years compared with the majority of Saudi Arabians whom their age less than 40 years. A previous study(Development and validation of a patient self-assessment score for diabetes risk) done by Bang et al. (2009) reported that, there was a strong correlation between type 2 diabetes mellitus and advanced age, especially after 40 years. In addition, Naglaa and Mohamed (2010) who studied the effectiveness of health education program for type 2 diabetes mellitus patients in Zagazig University diabetes clinic, reported highest percentage of diabetes patients were aged between (50-59) years old. Also, 35\% of patients who had uncontrolled type II diabetes were aged above 50 years old, the prevalence of diabetes raise with age to a maximum of $40 \%$ after the age of 55 years old.

Regarding gender distribution, these findings revealed that, two-thirds of Egyptians were female while more than two-thirds of Saudi Arabians were male. In contradiction with these findings a study by Naglaa and Mohamed (2010) which reported that, no significant changes were found between gender as their study found that less than half of diabetic patients were males and more than half were females.

Moreover, the results revealed that the $(58 \%$ versus $38 \%)$ of both groups have a positive family history of diabetes which supports the evidence of positive family history is a major risk in diabetes acquisition. Consistent with this result, another study carried out by El Bcheraoui et al. (2013) which study (Hypertension and Its Associated Risk Factors in the Kingdom of Saudi Arabia) reported that 121 diabetes patients out of 300 were had a positive family history of diabetes. While in contrast, a study by Saleh et al. (2012) which study (Knowledge and self-care practices regarding diabetes among newly diagnosed Type 2 diabetics in Bangladesh ) reported that, only non-significant number (29\%) were had a positive family history of diabetes mellitus in their study.

More than half of both groups had a positive history of hypertension .In support with us, the prevalence of hypertension are very high in Saudi Arabia about 40.6\% (El Bcheraoui et al., 2013). Furthermore, the Egyptian National hypertension Project reported that hypertension is common among Egyptians and $26.3 \%$ of adult Egyptians had high blood pressure. Also, more than $50 \%$ of individuals were suffered from hypertension according to study of Masanari et al., 2019 (Fasting blood glucose is predictive of hypertension in a general Japanese population) which reported that, at least 1 in 3 patients with diabetes type 1 also have hypertension.

Regarding body mass index the study findings revealed that in both groups the body mass index was above the normal with a non-significance difference which support the evidence of, overweight/obese extremely upsurges an individual's risk of developing other health problems as diabetes type II. Consistency to these findings, Alqarni (2016) found that Egypt has the highest percentage of obese adults worldwide around 19 million Egyptians which puts them at high risk of developing diabetes mellitus. Also, a previous study by Alahmed \& Lobelo (2018) revealed that the rate of obesity in Saudi Arabia significantly high among different age groups and occupations; at different places in the country and among both females and males which put them under the catastrophe of diabetes mellitus (Mahdi, 2013). The researcher guesses that in KSA the obesity may be due to diet and bad eating habits and the absence of physical activities with the modern sedentary life.

The last important risk factor shows a role in the current study findings was the level of physical activity between both groups and revealed that $50.7 \%$ versus $64 \%$ are physically active while $49.3 \%$ versus $36 \%$ are physically inactive respectively. From the researcher point of view, this is may be due to that the nature of work among the majority of both groups, which was mental, not muscular work.

Results also are well matched with a study about physical inactivity among Egyptian and Saudi medical students conducted by El-Gilany \& El-Masry (2015) which found physical inactivity significantly higher among Saudi Arabians than Egyptians. This is may be due to the lifestyle nature in Saudi Arabia completely different from the lifestyle in Egypt as the use of private cars for transportation.

Current results revealed that there were statistically significant differences as regarding total score of employee's knowledge and described that both groups had a good score of knowledge (74\% of Egyptians vs. 58\% of Saudi Arabians). In researcher view, the relatively higher rate of knowledgeable diabetics in the existing study can be explained by the higher percentage of educated members and the high rate of positive family history. Differing with our results, previous studies carried out by Naglaa \& Mohamed (2010) and Perez \& Cha (2017). They explained that the level of knowledge, attitude, and practice of diabetes were very poor in their studies.

Regarding the attitude toward diabetes, these study findings described a significant difference between both 
groups regarding total attitude score. Also, more than half of Egyptians had negative attitude compared to nearly half of Saudi Arabians who have had a positive attitude. This result can be clarified for Saudi Arabian's employee through their high level of education and a high score of knowledge regarding diabetes mellitus that may reflect positively on their attitude toward diabetes.

In the line of these findings study done by Saleh et al. (2012) who described a gap between knowledge on diabetes and attitudes toward diabetes. Even though the majority had either moderate or good knowledge, it is not mirrored on their attitudes and practices. From the researcher point of view, although it is difficult to realize the reasons for this gap, there may be a number of realistic reasons. One important reason is improper and uncoordinated health education. Nowadays most of the information get through social media. This might lead to a spread of information but in a way that is not convincing to the general population.

Lastly, the total score of employee's practices of both groups reported that, nearly two-thirds of Egyptians had good practice vs. $44 \%$ of Saudi Arabians who had a bad practice with statistically significant differences between both groups. This result reflects that knowledge does not constantly result in positive attitude and practices. Our results were in agreement with Salem et al. (2018) who studied the effect of diabetes mellitus knowledge on practice. The study revealed that, the participants comply with bad practice even though they were well aware of the deleterious effects of sugar on oral hygiene. In our opinion, poor practice among Saudi employees awing to poor physical activity, modern lifestyles like acceleration in car ownership, consumption of high-fat caloric dense food, refined sugar and salt, food habits and presence of house and nursery maid.

\section{Conclusion}

The study findings succeeded in answering both research questions. Based on the results of the current study, it was concluded that:

1. There were statistically significant differences among Egyptians versus Saudi Arabian's nursing employees in total knowledge, attitude and practice scores of diabetes mellitus.

2. Total risk assessment screening score of diabetes mellitus has shown statistically significant difference among Egyptians versus Saudi Arabian's nursing employees.

\section{Recommendations}

Based on the discoveries of the present study, the following recommendations can be suggested:

1. Large-scale awareness programs should be implemented after identifying the appropriate means to spread the message to the public population.

2. An innovative educational model should be developed to improve personnel attitudes and knowledge on all facets of diabetes.

3. Periodic and continuous diabetes mellitus screening should be planned by each country authorized personnel.

\section{References}

Alahmed, Z., and Lobelo, F. (2018). Physical activity promotion in Saudi Arabia: A critical role for clinicians and the health care system. Journal of Epidemiology and Global Health. https://doi.org/10.1016/j.jegh.2017.10.005.19.

Alqarni, S. (2016). A Review of Prevalence of Obesity in Saudi Arabia. Journal of Obesity \& Eating Disorders, 93 (1), S60-S65.

Bang, H., Edwards, A., Bomback, A., Ballantyne, C., Brillon D., Callaha, M., \& Kern L. (2009). Development and validation of a patient self-assessment score for diabetes risk. Annals of Internal Medicine. https://doi.org/10.1059/0003-4819-151-11-200912010-00005.

Barhum, L. (2017). How are diabetes and hypertension linked? Medical News Today.

Dawson, B. and Trapp, R. (2001). Reading the medical literature: Basic \& Clinical Biostatistics. Lange Medical Book/ McGraw - Hill. Medical Publication Division, New York. 3rd ed., Ch. 7-9, 161-218 and Ch. 13, PP 305-14.

Department of Health and Human Services, U.S. (2014). the Health Consequences of Smoking-50 Years of Progress: A Report of the Surgeon General. Atlanta, GA: U.S. Department of Health and Human Services, Centers for Disease Control and Prevention, National Center for Chronic Disease Prevention and Health Promotion, Office on Smoking and Health, 2014. Printed with corrections, January 2014.

El Bcheraoui, C., Memish, Z.A., Tuffaha, M., Daoud,F., Robinson, M., Jaber, S., Sarah Mikhitarian, S., Mohammad Al Saeedi, M., AlMazroa, M.A., Mokdad, A.H., and Al Rabeeah, A.A. (2013). Hypertension and Its Associated Risk Factors in the Kingdom of Saudi Arabia, 2013: A National Survey. International Journal of Hypertension, 2014, Article ID 564679, 8 pages, 2014. https://doi.org/10.1155/2014/564679.

El-Gilany, A., and El-Masry, R. (2015). Physical inactivity among Egyptian and Saudi medical students. TAF Preventive Medicine Bulletin, 10(1). 35-44 doi: 10.5455/pmb.20101018022143.

Farzana, S., Mumu, S., Ferdous, A., Begum, H., Ali, L. (2012). Knowledge and self-care practices regarding 
diabetes among newly diagnosed type 2 diabetics in Bangladesh: a cross-sectional study. BMC Public Health . $2012,12: 1112$.

Herath, H.M.M, Weerasinghe, N.P., Dias, H., and Weerarathna, T.P. (2017). Knowledge, attitude and practice related to diabetes mellitus among the general public in Galle district in Southern Sri Lanka: a pilot study. BMC Public Health, 17,535-541.

Kassahun, C. and Mekonen, A. (2017). Knowledge, attitude, practices and their associated factors towards diabetes mellitus among non-diabetes community members of Bale Zone administrative towns, South East Ethiopia. A cross-sectional study. PLoS ONE, 12(2), e0170040. https://doi.org/10.1371/journal.pone.0170040.

Mahdi, H., Hassan, Y., Bin Aziz, N., Abdulrazzaq, H., Jirjees, F., and Abduelkarem, A. (2013). Diabetes knowledge and practice in Malaysian and the United Arab Emirates diabetic patients. Research Journal of Pharmaceutical, Biological and Chemical Sciences. Journal of clinical and applied research and education, 9 (4).

Masanari, K., Chintaluru, Yaswanth, C., Kanbay, Mehmet, K., Niwa, Koichiro, N., Ichiro, H., Ana, A., Carlos, R., Minoru, O., Richard, J., Lanaspa, and Miguel, L.A..(2019). Fasting blood glucose is predictive of hypertension in a general Japanese population. Journal of Hypertension, 37 (1), 167-174.

Naeem, Z. (2015). Burden of Diabetes Mellitus in Saudi Arabia. International Journal of Health Sciences, 9 (3), V- VI.

Naglaa, A. and Mohamed, M. (2010), Effectiveness Of Health Education Program For Type 2 Diabetes Mellitus Patients Attending Zagazig University Diabetes Clinic, Egypt. Journal of Egyptian Public Health Association, $85(3 \& 4), 113-130$.

Perez, M. and Cha, K. (2017). Diabetes Knowledge, Beliefs, and Treatments in the Hmong Population: An Exploratory Study. Hmong Studies Journal, 8, 1-21.

Saleh, F., Mumu, S., Ara, F., Begum, H., and Ali, L. (2012): Knowledge and self-care practices regarding diabetes among newly diagnosed Type 2 diabetics in Bangladesh: A cross sectional study. BMC Public Health, 12, 1112 .

Salem, A., Majed, A., Abdulaziaz M., Mustafa, M., Abdulsalam, A.,Mohammed, S., Asdaq, B., Mohammed, A. (2018). Knowledge, Attitude, and Practice Regarding Diabetes Mellitus among General Public and Diabetic Patients in Riyadh, Saudi Arabia. Asian Journal of Pharmaceutics, 12 (1), S1.

Shulman G.I. (2014). Ectopic fat in insulin resistance, dyslipidemia, and cardiometabolic Disease. The New England Journal of Medicine, 371, 1131-41.

Whiting, D. R., Guariguata, L., Weil, C., and Shaw, J. (2011). IDF Diabetes Atlas: Global estimates of the prevalence of diabetes for 2011 and 2030. Diabetes Research and Clinical Practice, 94, 311- 321.

Yanling, W., Yanping, D., Yoshimasa, T., and Wen, Z. (2014). Risk factors contributing to type 2 diabetes and recent advances in the treatment and prevention. International journal of medical sciences, 11(11), 11851200.

Zhao, Y., Jiang, Z., Guo, C. (2011). Review New hope for type 2 diabetics: targeting insulin resistance through the immune modulation of stem cells. Autoimmunity Reviews, 11(2), 137-142. 